\title{
Thouless-Kohmoto-Nightingale-den Nijs formula for a general Hamiltonian
}

\author{
Hidenori Fukaya, ${ }^{*}$ Tetsuya Onogi, ${ }^{\dagger}$ and Satoshi Yamaguchi ${ }^{\ddagger}$ \\ Department of Physics, Osaka University, Toyonaka, Osaka 560-0043, Japan \\ $\mathrm{Xi} \mathrm{Wu}{ }^{\S}$ \\ Physics Department, Ariel University, Ariel
}

(Received 12 April 2019; accepted 25 March 2020; published 10 April 2020)

\begin{abstract}
Topological insulators in odd dimensions are characterized by topological numbers. We prove the wellknown relation between the topological number given by the Chern character of the Berry curvature and the Chern-Simons level of the low energy effective action for a general class of Hamiltonians bilinear in the fermion with general U(1) gauge interactions including nonminimal couplings by an explicit calculation. A series of Ward-Takahashi identities are crucial to relate the Chern-Simons level to a winding number, which could then be directly reduced to Chern character of Berry curvature by carrying out the integral over the temporal momenta.
\end{abstract}

DOI: $10.1103 /$ PhysRevD.101.074507

\section{INTRODUCTION}

Topological insulators in $D=2 n+1$ dimensions are characterized by topological numbers. One characterization is given by the Chern character of the Berry connection from the eigenfunctions of the Hamiltonian in the valence band [1,2], the other characterization is given by the level of the Chern-Simons action which appears in the effective action after integrating out the fermion coupled to a smooth external U(1) gauge field, i.e., photon [3-9]. These two characterizations are known to be equivalent because they both arise from the current correlation functions and there are explicit proofs for various cases.

For example, the famous Thouless-Kohmoto-Nightingaleden Nijs (TKNN) number [1] describes integer Hall conductivity in spatial two-dimensional systems and it is valid for all kinds of band structures neglecting interactions between electrons. Meanwhile, motivated by the discovery of the domain-wall fermion [10] (see also subsequent papers to study the anomaly inflow [11-13]) in their paper, Golterman, Jansen, and Kaplan also gave an expression of conductivity of Chern-Simons current for a wide class of fermion propagators on the lattice including Wilson fermion with odd-dimensional Euclidean spacetime and found out

\footnotetext{
*hfukaya@phys.sci.osaka-u.ac.jp

onogi@phys.sci.osaka-u.ac.jp

*yamaguch@ het.phys.sci.osaka-u.ac.jp

§wuxi5949@gmail.com
}

Published by the American Physical Society under the terms of the Creative Commons Attribution 4.0 International license. Further distribution of this work must maintain attribution to the author(s) and the published article's title, journal citation, and DOI. Funded by SCOAP ${ }^{3}$. that the topological number is given by the homotopy class for the map $T^{D} \rightarrow S^{D}$ [9]. They also showed that the topological number one finds correlated perfectly with the number of chiral edge states. The relation is well known to many people, and in the continuum theory or in some special models there exist studies for the relations $[14,15]$.

In Ref. [16] a proof is given for a large class of models for general odd dimensions, where they consider the most general lattice action for an arbitrary free kinetic term on the lattice which is then coupled to a U(1) gauge field in a minimal way, i.e., with the gauge interaction in the form of

$$
H(A)=\sum_{m, n} \psi_{m}^{\dagger} h_{m n} e^{i A_{m n}} \psi_{n}+\sum_{m} \psi_{m}^{\dagger} \psi_{m},
$$

where $m, n$ are the lattice sites, $h_{m n}$ are the hopping parameters, and $A_{m n}$ is the line integral of the gauge field along the straight line connecting the sites $m$ and $n$. The advantage of this class of Hamiltonian is that the contact interactions such as fermion-fermion-multiphoton vertices do not contribute to the final expression so that only a set of Feynman diagrams which appear also in the continuum theory gives nonvanishing contributions. Of course, this type of gauge interaction is physically motivated since it is based on the famous method of "Peierls substitution" [17]. However, in a more general situation, the gauge interaction may not always be described by such a single straight Wilson line. It could be a linear combination of various Wilson lines of arbitrary path, which can give nonminimal coupling. In such cases, one has to include the contribution of contact interaction vertices.

In this paper, we study the equivalence of the topological number from the TKNN formula and that from the 
Chern-Simons coupling for the most general lattice fermion Hamiltonian coupled to a $\mathrm{U}(1)$ gauge field which is bilinear in fermion. The new feature of our study is that the Hamiltonian is general enough to include arbitrary nonminimal gauge interactions, which has not been considered in the previous studies [3-9,14-16]. We give an explicit proof of the equivalence of the two topological numbers for gapped fermion systems with Hamiltonian on the lattice given by the bilinear form of the fermion coupled to external U(1) fermions. We also discuss how the relation can give the Chern character in a higher dimensional case.

The organization of this paper is as follows. In the next section we rewrite the level of the Chern-Simons effective action for the gapped fermion system coupled to a U(1) gauge field using the Feynman rule and relate it to the winding number of a map from $T^{D}$ to the fermion propagator space in $D=2+1$ and $D=4+1$ dimensions. In Sec. III, we show the equivalence of the winding number to the Chern number for the Berry curvature. Section IV is devoted to a summary and conclusion.

\section{GAPPED FERMION SYSTEM ON THE LATTICE}

\section{A. General gapped fermion system}

We consider a gapped fermion system on a lattice (or condensed matter systems on a translational invariant crystal) with the following action in Euclidean space in $D=2 n+1$ dimensions (note that the time is continuous but the space is discrete as in the condensed matter systems):

$$
S_{\mathrm{E}}=\int d t \sum_{\vec{r}} \psi^{\dagger}(t, \vec{r})\left[\frac{\partial}{\partial t}+i A_{0}+H(\vec{A})\right] \psi(t, \vec{r}),
$$

where $\vec{r}$ runs over the $2 n$ dimensional spatial lattice points. We will set $x^{0}=t$ in the following. The Hamiltonian $H(\vec{A})$ is given by a summation over all the possible hoppings on the lattice which include gauge interactions with a smooth external $\mathrm{U}(1)$ gauge field $A_{\mu}=\left(A_{0}, \vec{A}\right)$. The fermion fields $\psi^{\dagger}(t, \vec{r})$ and $\psi(t, \vec{r})$ give creation and annihilation operators of fermions after quantization. We assume that when the gauge field is turned off, the Hamiltonian is translational invariant so that it can allow band structures. We also assume that there are $N_{v}$ bands and $N_{c}$ bands below and above the Fermi level, respectively. Therefore the fermion fields have $N_{v}+N_{c}$ components.

\section{B. Effective gauge action}

Since the fermion system is gapped with a gap size $\Delta>0$, the effective gauge action obtained by integrating out fermions can be expanded in terms of gauge invariant local actions as

$$
S_{\text {eff }}=\sum_{k} a_{k} S_{k}(A)
$$

Here, $S_{\text {eff }}(A)$ is defined as

$$
e^{S_{\mathrm{eff}}}=\int \mathcal{D} \psi \mathcal{D} \psi^{\dagger} e^{-S_{\mathrm{E}}},
$$

and $S_{k}(A)$ are the gauge invariant actions given by the local Lagrangian $L_{k}(A)$ and $a_{k}$ are the coefficients. By dimensional analysis, if the Lagragian $L_{k}(A)$ has a mass dimension $d_{k}$, the coefficient $a_{k}$ is suppressed by the $d_{k}-(2 n+1)$ powers in $\frac{1}{\Delta}$ or lattice spacing $a$. Many of the Lagrangians are given in terms of a gauge invariant field $F_{\mu \nu}=\partial_{\mu} A_{\nu}-\partial_{\nu} A_{\mu}$ (e.g., $S_{\mu \nu, \rho \sigma}^{F^{2}} \equiv \int d^{2 n+1} r F_{\mu \nu} F_{\rho \sigma}$ with a coefficent $\left.a_{\mu \nu, \rho \sigma}^{F^{2}}\right)$. Since we do not have the Lorentzinvariance on the lattice, the structure of the coefficients $a_{k}$ in the effective action heavily depend on the geometry of the lattice.

However, there is a very special parity-violating term called Chern-Simons action $S_{\mathrm{cs}}(A)$ given by

$S_{\mathrm{cs}}(A)=\int d^{2 n+1} x \epsilon_{\alpha_{0} \beta_{1} \alpha_{1} \cdots \beta_{n} \alpha_{n}} A_{\alpha_{0}} \partial_{\beta_{1}} A_{\alpha_{1}} \cdots \partial_{\beta_{n}} A_{\alpha_{n}}$.

This action is topological and always takes this form no matter what the geometry of the lattice is. Topological information of the fermion system is contained in the effective action through the coefficient $c_{\mathrm{cs}}$ as

$$
S_{\text {eff }}(A)=i c_{\mathrm{cs}} S_{\mathrm{cs}}+\text { other gauge invariant terms. }
$$

Here the gauge invariance of the action requires that the coefficient is quantized as (see [14] for example)

$$
c_{\mathrm{cs}}=\frac{k}{(2 \pi)^{n}(n+1) !}, \quad k \in \mathbb{Z} .
$$

Since the Chern-Simons action is of the lowest dimension in the parity-violating sector, the coefficient $c_{\mathrm{cs}}$ can be obtained by the following quantity:

$$
c_{\mathrm{cs}}=\frac{(-i)^{n+1} \epsilon_{\alpha_{0} \beta_{1} \alpha_{1} \cdots \beta_{n} \alpha_{n}}}{(n+1) !(2 n+1) !}\left(\frac{\partial}{\partial\left(q_{1}\right)_{\beta_{1}}}\right) \cdots\left(\frac{\partial}{\partial\left(q_{n}\right)_{\beta_{n}}}\right) \times\left.\prod_{i=1}^{n} \int d^{D} x_{i} e^{i q_{i} x_{i}} \frac{\delta^{n+1} S_{\mathrm{eff}}(A)}{\delta A_{\alpha_{0}}\left(x_{0}\right) \delta A_{\alpha_{1}}\left(x_{1}\right) \cdots \delta A_{\alpha_{n}}\left(x_{n}\right)}\right|_{q_{i}=0} .
$$

In the continuum theory or in simple lattice fermions such as a Wilson fermion, this can be rewritten in terms of a fermion path integral as 


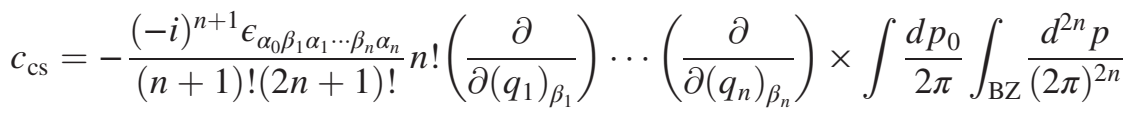

$$
\begin{aligned}
& \left.\operatorname{Tr}\left[S_{F}\left(p+q_{n+1}\right) \Gamma^{(1)}\left[q_{n+1}, \alpha_{0} ; p\right] S_{F}(p) \Gamma^{(1)}\left[q_{1}, \alpha_{1} ; p-q_{1}\right] S_{F}\left(p-q_{1}\right) \cdots \Gamma^{(1)}\left(q_{n}, \alpha_{n} ; p\right)\right]\right|_{q_{i}=0} .
\end{aligned}
$$

Here, BZ stands for the Brillioune zone, $q_{n+1} \equiv q_{1}+\cdots+$ $q_{n}, S_{F}(p)$ is the Fourier transform of the free fermion propagator $\frac{1}{\frac{\partial}{\partial x_{0}}+H(\vec{A}=0)}$ with momentum $p$, and $\Gamma^{(1)}[q, \alpha ; p]$ is the fermion-fermion-photon vertex with incoming fermion momentum $p$ and incoming photon momentum $q$. However, the situation is not simple in general, since there are also contributions from contact interactions such as fermion-fermion-multiphoton vertices, which can naturally arise from nonminimal gauge couplings or generic lattice artifacts. In the following, we will explicitly show that these contributions automatically cancel against the contributions from the momentum derivative of vertex functions due to the Ward-Takahashi identities.

In the next subsection, we formulate how to evaluate Eq. (8) using fermion propagators and vertex functions for a general Hamiltonian system.

\section{Fermion propagator representation of Eq. (8)}

The effective action can be given by the log of the fermion determinant as

$$
S_{\mathrm{eff}}(A)=\operatorname{Tr}\left[\ln \left(D_{0}+H(A)\right)\right]
$$

where $D_{0}=\frac{\partial}{\partial x^{0}}+i A_{0}$. Splitting the kinetic operator $D_{0}+H(A)$ into a free part and an interaction part reads as

$$
D_{0}+H(A)=\frac{\partial}{\partial x_{0}}+H_{0}-\Gamma(A)
$$

where $H_{0}$ is the free fermion part defined as $H_{0} \equiv$ $\left.H(A)\right|_{A=0}$ and $\Gamma(A)$ is the interaction part defined as $\Gamma(A) \equiv-i A_{0}-H(A)+H_{0}$. Plugging $\quad$ Eq. (11) into Eq. (10) we obtain

$$
S_{\text {eff }}(A)-\text { const. }=-\sum_{n=1}^{\infty} \frac{1}{n} \operatorname{Tr}\left[\left(\frac{1}{\frac{\partial}{\partial x_{0}}+H_{0}} \Gamma(A)\right)^{n}\right]
$$

Simple algebra shows that the following equation holds:

$$
\begin{aligned}
\left.\epsilon_{\alpha_{0} \beta_{1} \alpha_{1}} \frac{\delta^{2} S_{\text {eff }}}{\delta A_{\alpha_{0}}\left(x_{0}\right) \delta A_{\alpha_{1}}\left(x_{1}\right)}\right|_{A=0}= & -\epsilon_{\alpha_{0} \beta_{1} \alpha_{1}}\left\{\left.\operatorname{Tr}\left[\frac{1}{\frac{\partial}{\partial x_{0}}+H_{0}} \cdot \frac{\delta^{2} \Gamma(A)}{\delta A_{\alpha_{0}}\left(x_{0}\right) \delta A_{\alpha_{1}}\left(x_{1}\right)}\right]\right|_{A=0}\right. \\
& \left.+\left.\operatorname{Tr}\left[\frac{1}{\frac{\partial}{\partial x_{0}}+H_{0}} \cdot \frac{\delta \Gamma(A)}{\delta A_{\alpha_{0}}\left(x_{0}\right)} \cdot \frac{1}{\frac{\partial}{\partial x_{0}}+H_{0}} \cdot \frac{\delta \Gamma(A)}{\delta A_{\alpha_{1}}\left(x_{1}\right)}\right]\right|_{A=0}\right\} .
\end{aligned}
$$

From Eq. (13), we find that the Chern-Simons coupling for $D=2+1$ dimensions is given by

$$
\begin{aligned}
c_{\mathrm{cs}}= & -\frac{(-i)^{2} \epsilon_{\alpha_{0} \beta_{1} \alpha_{1}}}{2 ! 3 !} \int \frac{d^{3} p}{(2 \pi)^{3}}\left(\frac{\partial}{\partial q_{1}}\right)_{\beta_{1}}\left\{\operatorname{Tr}\left[S_{F}(p) \Gamma^{(2)}\left[-q_{1}, \alpha_{0} ; q_{1}, \alpha_{1} ; p\right]\right]\right. \\
& \left.+\operatorname{Tr}\left[S_{F}\left(p-q_{1}\right) \Gamma^{(1)}\left[-q_{1}, \alpha_{0} ; p\right] S_{F}(p) \Gamma^{(1)}\left[q_{1}, \alpha_{1} ; p-q_{1}\right]\right]\right\}\left.\right|_{q_{1}=0},
\end{aligned}
$$

where $S_{F}(p)$ is the fermion propagator $\frac{1}{i p_{0}+H_{0}(\vec{p})}$ and $\Gamma^{(1)}\left[q_{1}, \alpha_{1} ; p\right]$ and $\Gamma^{(2)}\left[q_{1}, \alpha_{1} ; q_{2}, \alpha_{2} ; p\right]$ are fermion-fermion-photon and fermion-fermion-photon-photon vertices with incoming fermion momentum $p$ and incoming photon momenta $q_{i}$ $(i=1,2)$ with Lorentz index $\alpha_{i}(i=1,2)$

$$
\begin{gathered}
\Gamma^{(1)}\left[q_{1}, \alpha_{1} ; p\right]=\left.\int d^{2 n+1} x_{1} e^{i q_{1} \cdot x_{1}} \int d^{2 n+1} y e^{i p \cdot y} \frac{\delta \Gamma[A](x, y)}{\delta A_{\alpha_{1}}\left(x_{1}\right)}\right|_{A=0, x=0}, \\
\Gamma^{(2)}\left[q_{1}, \alpha_{1}, q_{2} ; \alpha_{2} ; p\right]=\left.\prod_{i=1}^{2}\left(\int d^{2 n+1} x_{i} e^{i q_{i} \cdot x_{i}}\right) \int d^{2 n+1} y e^{i p \cdot y} \frac{\delta^{2} \Gamma[A](x, y)}{\delta A_{\alpha_{1}}\left(x_{1}\right) \delta A_{\alpha_{2}}\left(x_{2}\right)}\right|_{A=0, x=0} .
\end{gathered}
$$

Note that the contributions with multiphoton vertices vanish for the class of Hamiltonians with gauge interactions given by a single straight Wilson line because the multiphoton vertices are symmetric under the interchange of Lorentz indices of 
photons. When contracted with the antisymmetric tensor, such contributions vanish. However, in general Hamiltonian we must consider these contributions.

From a similar calculation, we find that the Chern-Simons level for $D=4+1$ dimensions is given by

$$
\begin{aligned}
c_{\mathrm{cs}}= & -\frac{(-i)^{3} \epsilon_{\alpha_{0} \beta_{1} \alpha_{1} \beta_{2} \alpha_{2}}}{3 ! 5 !} \int \frac{d^{5} p}{(2 \pi)^{5}}\left(\frac{\partial}{\partial q_{1}}\right)_{\beta_{1}}\left(\frac{\partial}{\partial q_{2}}\right)_{\beta_{2}}\left\{\operatorname{Tr}\left[S_{F}(p) \cdot \Gamma^{(3)}\left[-\left(q_{1}+q_{2}\right), \alpha_{0} ; q_{1}, \alpha_{1} ; q_{2}, \alpha_{2} ; p\right]\right]\right. \\
& +2 \operatorname{Tr}\left[S_{F}\left(p-q_{2}\right) \Gamma^{(2)}\left[-\left(q_{1}+q_{2}\right), \alpha_{0} ; q_{1}, \alpha_{1} ; p\right] S_{F}(p) \Gamma^{(1)}\left[q_{2}, \alpha_{2} ; p-q_{2}\right]\right] \\
& \left.+\operatorname{Tr}\left[S_{F}\left(p+q_{1}+q_{2}\right) \Gamma^{(2)}\left[q_{1}, \alpha_{1} ; q_{2}, \alpha_{2} ; p\right] S_{F}(p) \Gamma^{(1)}\left[-\left(q_{1}+q_{2}\right), \alpha_{0} ; p+q_{1}+q_{2}\right]\right]\right\}, \\
& \left.+2 \operatorname{Tr}\left[S_{F}\left(p+q_{1}\right) \Gamma^{(1)}\left[q_{1}, \alpha_{1} ; p\right] S_{F}(p) \Gamma^{(1)}\left[q_{2}, \alpha_{2} ; p-q_{2}\right] S_{F}\left(p-q_{2}\right) \Gamma^{(1)}\left[-\left(q_{1}+q_{2}\right), \alpha_{0} ; p+q_{1}\right]\right]\right\}\left.\right|_{q_{1}=q_{2}=0},
\end{aligned}
$$

where $\Gamma^{(3)}\left[q_{1}, \alpha_{1} ; q_{2}, \alpha_{2} ; q_{3}, \alpha_{3} ; p\right]$ is the fermion-fermion-photon-photon-photon vertex with incoming fermion momentum $p$ and incoming photon momenta $q_{i}(i=1,2,3)$ with Lorentz index $\alpha_{i}(i=1,2,3)$, which is given by

$$
\Gamma^{(3)}\left[q_{1}, \alpha_{1}, q_{2} ; \alpha_{2} \cdot q_{3}, \alpha_{3} ; p\right]=\left.\prod_{i=1}^{3}\left(\int d^{2 n+1} x_{i} e^{i q_{i} \cdot x_{i}}\right) \int d^{2 n+1} y e^{i p \cdot y} \frac{\delta^{3} \Gamma[A](x, y)}{\left.\delta A_{\alpha_{1}}\left(x_{1}\right) \delta A_{\alpha_{2}\left(x_{2}\right)} \delta A_{\alpha_{3}\left(x_{3}\right)}\right)}\right|_{A=0, x=0} .
$$

\section{The case for $D=2+1$ dimensions $(n=1)$}

In $D=2+1$ dimensions, i.e., the $n=1$ case, the Chern-Simons coupling $c_{\mathrm{cs}}$ has contributions from the loop involving two fermion-fermion-photon vertices and the loop involving a single fermion-fermion-photon-photon vertex (contact interaction) as

$$
c_{\mathrm{cs}}=-\frac{(-i)^{2}}{2 ! 3 !} \int \frac{d^{3} p}{(2 \pi)^{3}} X
$$

where $X$ is given as

$X=\epsilon_{\alpha_{0} \beta_{1} \alpha_{1}}\left(\frac{\partial}{\partial q_{1}}\right)_{\beta_{1}}\left\{\operatorname{Tr}\left[S_{F}(p) \Gamma^{(2)}\left[-q_{1}, \alpha_{0} ; q_{1}, \alpha_{1} ; p\right]\right]+\operatorname{Tr}\left[S_{F}\left(p-q_{1}\right) \Gamma^{(1)}\left[-q_{1}, \alpha_{0} ; p\right] S_{F}(p) \Gamma^{(1)}\left[q_{1}, \alpha_{1} ; p-q_{1}\right]\right]\right\}$.

The first term on the right-hand side is the one-loop contribution with contact interaction and the second term is the usual one-loop contribution with simple fermion-fermion-photon vertices.

Carrying out the momentum derivative with $q$,

$$
\begin{aligned}
X= & \epsilon_{\alpha_{0} \beta_{1} \alpha_{1}} \times\left\{\left.\operatorname{Tr}\left[S_{F}(p)\left(-\frac{\partial \Gamma^{(2)}\left[q, \alpha_{0} ; 0, \alpha_{1} ; p\right]}{\partial q_{\beta_{1}}}+\frac{\partial \Gamma^{(2)}\left[0, \alpha_{0} ; q, \alpha_{1} ; p\right]}{\partial q_{\beta_{1}}}\right)\right]\right|_{q=0}\right. \\
& +\left.\operatorname{Tr}\left[S_{F}(p)\left(\frac{\partial \Gamma^{(1)}\left[q, \alpha_{1} ; p\right]}{\partial q_{\beta_{1}}}-\frac{\partial \Gamma^{(1)}\left[0, \alpha_{1} ; p\right]}{\partial p_{\beta_{1}}}\right) S_{F}(p) \Gamma^{(1)}\left(0, \alpha_{0} ; p\right)\right]\right|_{q=0} \\
& -\left.\operatorname{Tr}\left[S_{F}(p) \Gamma^{(1)}\left[0, \alpha_{1} ; p\right] S_{F}(p) \frac{\partial \Gamma^{(1)}\left(q, \alpha_{0} ; p\right)}{\partial q_{\beta_{1}}}\right]\right|_{q=0} \\
& \left.-\left.\operatorname{Tr}\left[S_{F}(p) \Gamma^{(1)}\left[0, \alpha_{1} ; p\right] \frac{\partial S_{F}(p)}{\partial p_{\beta_{1}}} \Gamma^{(1)}\left(0, \alpha_{0} ; p\right)\right]\right|_{q=0}\right\} .
\end{aligned}
$$

In Appendix A, we derive the Ward-Takahashi identities as follows:

$$
\Gamma^{(1)}[0, \alpha ; p]=-\frac{\partial S_{F}^{-1}(p)}{\partial p_{\alpha}}
$$




$$
\left.\frac{\partial^{2} \Gamma^{(1)}[k, \mu ; p]}{\partial k_{\nu} \partial p_{\lambda}}\right|_{k=0}=\left.\frac{\partial \Gamma^{(2)}[k, \mu ; 0, \lambda ; p]}{\partial k_{\nu}}\right|_{k=0}=\left.\frac{\partial \Gamma^{(2)}[0, \lambda ; l, \mu ; p]}{\partial l_{\nu}}\right|_{l=0} .
$$

Using these identities, we obtain

$$
\begin{aligned}
X= & \epsilon_{\alpha_{0} \beta_{1} \alpha_{1}} \times\left\{\left.2 \operatorname{Tr}\left[S_{F}(p)\left(\frac{\partial^{2} \Gamma^{(1)}\left[q, \alpha_{1} ; p\right]}{\partial q_{\beta_{1}} \partial p_{\alpha_{0}}}\right)\right]\right|_{q=0}+\left.2 \operatorname{Tr}\left[\frac{\partial S_{F}(p)}{\partial p_{\alpha_{0}}} \frac{\partial \Gamma^{(1)}\left[q, \alpha_{1} ; p\right]}{\partial q_{\beta_{1}}}\right]\right|_{q=0}\right. \\
& \left.+\left.\operatorname{Tr}\left[S_{F}(p) \frac{\partial S_{F}^{-1}(p)}{\partial p_{\alpha_{1}}} S_{F}(p) \frac{\partial S_{F}^{-1}(p)}{\partial p_{\beta_{1}}} S_{F}(p) \frac{\partial S_{F}^{-1}(p)}{\partial p_{\alpha_{0}}}\right]\right|_{q=0}\right\} .
\end{aligned}
$$

The first and the second terms on the right-hand side can be combined to give a total divergence which vanishes when we integrate over the momentum. Therefore, one finds that the Chern-Simons coupling is given by the winding number as

$$
c_{\mathrm{cs}}=\frac{(-i)^{2} \epsilon_{\alpha_{0} \beta_{1} \alpha_{1}}}{2 ! 3 !} \int \frac{d p_{0}}{2 \pi} \int_{\mathrm{BZ}} \frac{d^{2} p}{(2 \pi)^{2}} \operatorname{Tr}\left[S_{F}(p) \frac{\partial S_{F}^{-1}(p)}{\partial p_{\alpha_{0}}} S_{F}(p) \frac{\partial S_{F}^{-1}(p)}{\partial p_{\beta_{1}}} S_{F}(p) \frac{\partial S_{F}^{-1}(p)}{\partial p_{\alpha_{1}}}\right] .
$$

\section{E. The case for $D=4+1$ dimensions $(n=2)$}

The Chern-Simons coupling can be written as

$$
c_{\mathrm{cs}}=-\frac{(-i)^{3}}{3 ! 5 !} \int \frac{d^{5} p}{(2 \pi)^{5}}\left[X_{1}+X_{2}+X_{3}+X_{4}\right]
$$

where $X_{1}, X_{2}, X_{3}, X_{4}$ are defined as follows:

$$
\begin{aligned}
X_{1}= & \left.\epsilon_{\alpha_{0} \beta_{1} \alpha_{1} \beta_{2} \alpha_{2}}\left(\frac{\partial}{\partial q_{1}}\right)_{\beta_{1}}\left(\frac{\partial}{\partial q_{2}}\right)_{\beta_{2}}\left\{\operatorname{Tr}\left[S_{F}(p) \cdot \Gamma^{(3)}\left[-\left(q_{1}+q_{2}\right), \alpha_{0} ; q_{1}, \alpha_{1} ; q_{2}, \alpha_{2} ; p\right]\right]\right\}\right|_{q_{1}=q_{2}=0}, \\
X_{2}= & \left.\epsilon_{\alpha_{0} \beta_{1} \alpha_{1} \beta_{2} \alpha_{2}}\left(\frac{\partial}{\partial q_{1}}\right)_{\beta_{1}}\left(\frac{\partial}{\partial q_{2}}\right)_{\beta_{2}}\left\{2 \operatorname{Tr}\left[S_{F}\left(p-q_{2}\right) \Gamma^{(2)}\left[-\left(q_{1}+q_{2}\right), \alpha_{0} ; q_{1}, \alpha_{1} ; p\right] S_{F}(p) \Gamma^{(1)}\left[q_{2}, \alpha_{2} ; p-q_{2}\right]\right]\right\}\right|_{q_{1}=q_{2}=0}, \\
X_{3}= & \left.\epsilon_{\alpha_{0} \beta_{1} \alpha_{1} \beta_{2} \alpha_{2}}\left(\frac{\partial}{\partial q_{1}}\right)_{\beta_{1}}\left(\frac{\partial}{\partial q_{2}}\right)_{\beta_{2}}\left\{\operatorname{Tr}\left[S_{F}\left(p+q_{1}+q_{2}\right) \Gamma^{(2)}\left[q_{1}, \alpha_{1} ; q_{2}, \alpha_{2} ; p\right] S_{F}(p) \Gamma^{(1)}\left[-\left(q_{1}+q_{2}\right), \alpha_{0} ; p+q_{1}+q_{2}\right]\right]\right\}\right|_{q_{1}=q_{2}=0}, \\
X_{4}= & \epsilon_{\alpha_{0} \beta_{1} \alpha_{1} \beta_{2} \alpha_{2}}\left(\frac{\partial}{\partial q_{1}}\right)_{\beta_{1}}\left(\frac{\partial}{\partial q_{2}}\right)_{\beta_{2}}\left\{2 \operatorname { T r } \left[S_{F}\left(p+q_{1}\right) \Gamma^{(1)}\left[q_{1}, \alpha_{1} ; p\right] S_{F}(p) \Gamma^{(1)}\left[q_{2}, \alpha_{2} ; p-q_{2}\right] S_{F}\left(p-q_{2}\right)\right.\right. \\
& \left.\left.\times \Gamma^{(1)}\left[-\left(q_{1}+q_{2}\right), \alpha_{0} ; p+q_{1}\right]\right]\right\}\left.\right|_{q_{1}=q_{2}=0} .
\end{aligned}
$$

Using Eqs. (22), (23) as in the case of $D=2+1$ dimensions. as well as the following Ward-Takahashi identities given in Appendix A,

$$
\left.\frac{\partial^{2} \Gamma^{(3)}[q, \mu ; r, \nu ; 0, \lambda ; p]}{\partial q_{\alpha} \partial r_{\beta}}\right|_{q=r=0}=\left.\frac{\partial^{3} \Gamma^{(2)}[q, \mu ; r, \nu ; p]}{\partial q_{\alpha} \partial r_{\beta} \partial p_{\lambda}}\right|_{q=r=0}
$$

we obtain 


$$
\begin{aligned}
X_{1}+X_{2}+X_{3}+X_{4}= & \epsilon_{\alpha_{0} \beta_{1} \alpha_{1} \beta_{2} \alpha_{2}}\left[\frac { \partial } { \partial p _ { \alpha _ { 0 } } } \left\{3 \operatorname{Tr}\left[\left.\frac{\Gamma^{(2)}\left[q, \alpha_{1} ; r, \alpha_{2} ; p\right]}{\partial q_{\beta_{1}} r_{\beta_{2}}}\right|_{q=r=0} S_{F}(p)\right]\right.\right. \\
& +3 \operatorname{Tr}\left[\left.\left.\frac{\Gamma^{(1)}\left[q, \alpha_{1} ; p\right]}{\partial q_{\beta_{1}}}\right|_{q=0} S_{F}(p) \frac{\Gamma^{(1)}\left[q^{\prime}, \alpha_{2} ; p\right]}{\partial q_{\beta_{2}}^{\prime}}\right|_{q^{\prime}=0} S_{F}(p)\right] \\
& \left.+8 \operatorname{Tr}\left[\left.\frac{\Gamma^{(1)}\left[q, \alpha_{1} ; p\right]}{\partial q_{\beta_{1}}}\right|_{q=r=0} S_{F}(p) \frac{\partial S_{F}^{-1}(p)}{\partial p_{\alpha_{2}}} \frac{\partial S_{F}(p)}{\partial p_{\beta_{2}}}\right]\right\} \\
& \left.+2 \operatorname{Tr}\left[S_{F}(p) \frac{\partial S_{F}^{-1}(p)}{\partial p_{\alpha_{0}}} S_{F}(p) \frac{\partial S_{F}^{-1}(p)}{\partial p_{\beta_{1}}} S_{F}(p) \frac{\partial S_{F}^{-1}(p)}{\partial p_{\alpha_{1}}} S_{F}(p) \frac{\partial S_{F}^{-1}(p)}{\partial p_{\beta_{2}}} S_{F}(p) \frac{\partial S_{F}^{-1}(p)}{\partial p_{\alpha_{2}}}\right]\right] .
\end{aligned}
$$

The total divergence term will vanish after integrating over the spatial momenta due to the periodicity in BZ.

Thus, we finally get

$c_{\mathrm{cs}}=-\frac{(-i)^{3} \cdot 2}{3 ! 5 !} \int \frac{d^{5} p}{(2 \pi)^{5}} \epsilon_{\alpha_{0} \beta_{1} \alpha_{1} \beta_{2} \alpha_{2}} \operatorname{Tr}\left[S_{F}(p) \frac{\partial S_{F}^{-1}(p)}{\partial p_{\alpha_{0}}} S_{F}(p) \frac{\partial S_{F}^{-1}(p)}{\partial p_{\beta_{1}}} S_{F}(p) \frac{\partial S_{F}^{-1}(p)}{\partial p_{\alpha_{1}}} S_{F}(p) \frac{\partial S_{F}^{-1}(p)}{\partial p_{\beta_{2}}} S_{F}(p) \frac{\partial S_{F}^{-1}(p)}{\partial p_{\alpha_{2}}}\right]$.

Therefore, the Chern-Simons coupling is given by the winding number with fermion propagator also for the $D=4+1$ case.

We expect that the relation of a Chern-Simons coupling and the winding number for a general Hamiltonian including nonminimal coupling holds for arbitrary odd dimensions $(D=2 n+1)$. This will be left for future studies.

\section{EQUIVALENCE OF A WINDING NUMBER AND A CHERN NUMBER}

In this section, we show the equivalence of the Chern-Simons coupling given by the winding number expression and the Chern character given by the Berry connection for the energy eigenstates in the valence bands.

The proof of this part is already given in Ref. [16], but since the proof is simple, we give it here for completeness. We give the calculation for arbitrary odd $(D=2 n+1)$ dimensions, even though we have shown that the Chern-Simons coupling $c_{\mathrm{cs}}$ can be written by the winding number using $S_{F}$ only for $D=2+1$ and $D=4+1$ dimensions.

In order to simplify the notation, hereafter we abbreviate the derivative with respect to the momentum $p_{\mu}$ as $\partial_{\mu} \equiv \frac{\partial}{\partial p_{\mu}}$.

\section{A. Winding number in $D=\mathbf{2 n}+\mathbf{1}$ dimensions}

The result of the previous section for $D=2+1$ and $D=4+1$ can be unified to the following results: In the expression using the fermion propagator $S(p)$, the Chern-Simons coupling $c_{\mathrm{cs}}$ in $D=2 n+1$ dimensions is given as

$$
\begin{aligned}
c_{\mathrm{cs}} & =-\frac{n ! \cdot(2 n+1)(-i)^{n+1} \epsilon^{i_{1} i_{2} \cdots i_{2 n}}}{(n+1) !(2 n+1) !} \int \frac{d^{2 n} p}{(2 \pi)^{2 n}} \int \frac{d p^{0}}{2 \pi} \operatorname{Tr}\left[S_{F}\left(\partial_{0} S_{F}^{-1}\right) \prod_{k=1}^{2 n}\left(S_{F}\left(\partial_{i_{k}} S_{F}^{-1}\right)\right)\right] \\
& =-\frac{n ! \cdot(2 n+1)(-i)^{n+1} \epsilon^{i_{1} i_{2} \cdots i_{2 n}}}{(n+1) !(2 n+1) !} \int \frac{d^{2 n} p}{(2 \pi)^{2 n}} \int \frac{d p^{0}}{2 \pi} \operatorname{Tr}\left[\frac{1}{i p^{0}+H} i \prod_{k=1}^{2 n}\left(\frac{1}{i p^{0}+H}\left(\partial_{i_{k}} H\right)\right)\right] .
\end{aligned}
$$

Next we insert a complete set $\sum_{\alpha}|\alpha\rangle\langle\alpha|$, where $\alpha$ is the label of energy. Then we have

$$
c_{\mathrm{cs}}=\frac{n !(-i)^{n+2}}{(n+1) !(2 n) !} \int \frac{d^{2 n} p}{(2 \pi)^{2 n}} J .
$$

Here $J$ is defined as

$$
J=\sum_{\alpha_{1}, \ldots, \alpha_{2 n}} \epsilon^{i_{1} i_{2} \cdots i_{2 n}} \int \frac{d p^{0}}{2 \pi} \frac{\left\langle\alpha_{1}\left|\partial_{i_{1}} H\right| \alpha_{2}\right\rangle\left\langle\alpha_{2}\left|\partial_{i_{2}} H\right| \alpha_{3}\right\rangle \cdots\left\langle\alpha_{2 n}\left|\partial_{i_{2 n}} H\right| \alpha_{1}\right\rangle}{\left(i p^{0}+E_{\alpha_{1}}\right)^{2}\left(i p^{0}+E_{\alpha_{2}}\right) \cdots\left(i p^{0}+E_{\alpha_{2 n}}\right)}
$$


where $i_{1}, \ldots, i_{2 n}$ stands for the spatial indices and summation over these indices are implicitly assumed following Einstein's contraction rule. All we have to do is to integrate over $p^{0}$ using Cauchy's theorem. In order to discuss it in detail let us define the key integral $J$ as follows.

\section{B. $p^{0}$ integration}

Here, we use a trick to simplify the integration. It is easy to see that the expression Eq. (31) is invariant under continuous deformation of $S_{F}$ (or $H$ ) provided that the integrand remains to have no singularities. Therefore, under a continuous change of the Hamilitonian, the winding number remains unchanged from its original value as long as the energy spectrum is kept gapped throughout the deformation.

Now, the most general Hamiltonian with $N_{v}$ valence bands and $N_{c}$ conduction bands is expressed as

$H(\vec{p}) \equiv \sum_{a=1}^{N_{v}} E_{a}(\vec{p})|a(\vec{p})\rangle\left\langle a(\vec{p})\left|+\sum_{\dot{b}=1}^{N_{c}} E_{\dot{b}}(\vec{p})\right| \dot{b}(\vec{p})\right\rangle\langle\dot{b}(\vec{p})|$,

where $|a(\vec{p})\rangle$ labeled by $a$ is the energy eigenstate in the valence band with spatial momentum $\vec{p}$ and negative energy eigenvalue $E_{a}(\vec{p})<0$. The state $|\dot{b}(\vec{p})\rangle$ labeled by $\dot{b}$ is the energy eigenstate in the conduction band with spatial momentum $\vec{p}$ and positive energy eigenvalue $E_{\dot{b}}(\vec{p})>0$. One can continuously deform the Hamiltonian without hitting the singularity of $S(p)$ (i.e., keeping the system gapped) so that all energy eigenvalues in the conduction bands and all energy eigenvalues in the valence bands are degenerate and momentum independent (i.e., flat band), respectively.

Then the deformed Hamiltonian $H_{\text {new }}$ which gives the same winding number becomes

$H_{\text {new }}(\vec{p})=E_{v} \sum_{a=1}^{N_{v}}|a(\vec{p})\rangle\left\langle a(\vec{p})\left|+E_{c} \sum_{\dot{b}=1}^{N_{c}}\right| \dot{b}(\vec{p})\right\rangle\langle\dot{b}(\vec{p})|$,

where $E_{v}<0, E_{c}>0$ are the momentum independent constant. Here the eigenstates are identical to those with the original Hamiltonian.

Using Eqs. (B9) and (B10), one finds that in the insertion of eigenstates sandwiching $\partial_{i} H$, if states in the conduction bands appear in a row or if states in the valence bands appear in a row, the matrix element vanishes. Therefore, in Eq. (33) states in the valence bands and the states in the conduction bands should appear in an alternating order. Therefore, $J$ is expressed as

$$
\begin{aligned}
J= & \sum_{a_{1}, \ldots, a_{n}=1}^{N_{v}} \sum_{\dot{a}_{1}, \ldots, \dot{a}_{n}=1}^{N_{c}} \epsilon^{i_{1} j_{1} \cdots i_{2 n} j_{2 n}} \\
& {\left[\int \frac{d p^{0}}{2 \pi} \frac{1}{\left(i p^{0}+E_{v}\right)^{n+1}\left(i p^{0}+E_{c}\right)^{n}}\left\langle a_{1}\left|\partial_{i_{1}} H\right| \dot{a}_{1}\right\rangle\left\langle\dot{a}_{1}\left|\partial_{j_{1}} H\right| a_{2}\right\rangle \times \cdots \times\left\langle a_{n}\left|\partial_{i_{n}} H\right| \dot{a}_{n}\right\rangle\left\langle\dot{a}_{n}\left|\partial_{j_{n}} H\right| a_{1}\right\rangle\right.} \\
& \left.+\int \frac{d p^{0}}{2 \pi} \frac{1}{\left(i p^{0}+E_{c}\right)^{n+1}\left(i p^{0}+E_{v}\right)^{n}}\left\langle\dot{a}_{1}\left|\partial_{i_{1}} H\right| a_{1}\right\rangle\left\langle a_{1}\left|\partial_{j_{1}} H\right| \dot{a}_{2}\right\rangle \times \cdots \times\left\langle\dot{a}_{n}\left|\partial_{i_{n}} H\right| a_{n}\right\rangle\left\langle a_{n}\left|\partial_{j_{n}} H\right| \dot{a}_{1}\right\rangle\right] .
\end{aligned}
$$

Renaming the labels for eigenstates and using the definition of the master integral in Appendix $\mathrm{C}$ and substituting Eqs. (B11) and (B12), we have

$$
\begin{aligned}
J= & \sum_{a_{1}, \ldots, a_{n}=1}^{N_{v}} \sum_{\dot{a}_{1}, \ldots, \dot{a}_{n}=1}^{N_{c}} \epsilon^{i_{1} j_{1} \cdots i_{2 n} j_{2 n}}(-1)^{n}\left(E_{c}-E_{v}\right)^{2 n}\left(I^{[n+1, n]}\left(E_{v}, E_{c}\right)-I^{[n+1, n]}\left(E_{c}, E_{v}\right)\right) \\
& \left\langle a_{1} \mid \partial_{i_{1}} \dot{a}_{1}\right\rangle\left\langle\dot{a}_{1} \mid \partial_{j_{1}} a_{2}\right\rangle \times \cdots \times\left\langle a_{n} \mid \partial_{i_{n}} \dot{a}_{n}\right\rangle\left\langle\dot{a}_{n} \mid \partial_{j_{n}} a_{1}\right\rangle,
\end{aligned}
$$

where $I^{[m, n]}\left(E_{1}, E_{2}\right)$ is defined as

$$
I^{[m, n]}\left(E_{1}, E_{2}\right) \equiv \int \frac{d p^{0}}{2 \pi} \frac{1}{\left(i p^{0}+E_{1}\right)^{m}\left(i p^{0}+E_{2}\right)^{n}} .
$$

The expression of $I^{[m, n]}\left(E_{1}, E_{2}\right)$ after $p^{0}$ integration is given in Appendix C.

Substituting Eq. (C3) into Eq. (37), we obtain

$$
J=-\sum_{a_{1}, \ldots, a_{n}=1}^{N_{v}} \sum_{\dot{a}_{1}, \ldots, \dot{a}_{n}=1}^{N_{c}} \epsilon^{i_{1} j_{1} \cdots i_{2 n} j_{2 n}} \frac{(2 n) !}{(n !)^{2}}\left\langle a_{1} \mid \partial_{i_{1}} \dot{a}_{1}\right\rangle\left\langle\dot{a}_{1} \mid \partial_{j_{1}} a_{2}\right\rangle \times \cdots \times\left\langle a_{n} \mid \partial_{i_{n}} \dot{a}_{n}\right\rangle\left\langle\dot{a}_{n} \mid \partial_{j_{n}} a_{1}\right\rangle
$$


Using the formula for the Berry curvature in Eq. (B14), $J$ is finally expressed as

$$
\begin{aligned}
J= & (-1)^{n+1} \sum_{a_{1}, \ldots, a_{n}=1}^{N_{v}} \sum_{\dot{a}_{1}, \ldots, \dot{a}_{n}=1}^{N_{c}} \epsilon^{i_{1} j_{1} \cdots i_{2 n} j_{2 n}} \\
& \times \frac{(2 n) !}{(n !)^{2}} i^{n} \mathcal{F}_{i_{1} j_{1}}^{a_{1} a_{2}} \ldots \mathcal{F}_{i_{n} j_{n}}^{a_{n} a_{n+1}} .
\end{aligned}
$$

\section{Results of $c_{\mathrm{cs}}$}

Plugging Eq. (40) into Eq. (32)

$$
c_{\mathrm{cs}}=\frac{(-1)^{n}}{(n+1) !(2 \pi)^{n}} \int_{B Z} \operatorname{ch}_{n}(\mathcal{A}),
$$

where $\operatorname{ch}_{n}(\mathcal{A})$ is the second Chern character defined by

$$
\mathrm{ch}_{n}(\mathcal{A})=\frac{1}{n !} \frac{1}{(2 \pi)^{n}} \operatorname{tr}\left(\mathcal{F}^{n}\right) .
$$

Comparing this expression with Eq. (7)

$$
c_{\mathrm{cs}}=\frac{k}{(n+1) !(2 \pi)^{n}},
$$

we arrive at the relation

$$
k=(-1)^{n} \int_{B Z} \operatorname{ch}_{n}(\mathcal{A}) .
$$

This means that the Chern-Simons level and the topological number in terms of the Berry connection is shown to be identical.

\section{SUMMARY AND CONCLUSION}

We derived a general TKNN formula from a ChernSimons level in the effective action for a lattice system with a general Hamiltonian bilinear in fermions in $(2+1)$ and $(4+1)$ dimensions. We have shown that the ChernSimons level is given by the winding number of a map from $T^{D}$ to the fermion propagator space. For this relation, Ward-Takahashi identities including higher order relations are crucial.

There has been an understanding that for the field theory approach to work, there should be a low energy mode which can be described by a relativistic field theory. Therefore, the impression was that the field theory approach works only with a special type of system with emergent relativistic spectrum. The interesting point to note in our proof is that one does not need to assume anything but gauge invariance. The detailed structure of minimal or nonminimal gauge coupling is irrelevant. Also one does not need to assume that there exists an effective theory described by the relativistic field theory and it applies to any system including arbitrary bands which may be far away from the Fermi level.

Since we have found that the two methods can equally work well, we are now certain that we can use field theory approach to study the topological properties for arbitrary condensed matter systems which include interactions where one can fully utilize the power of field theory.

There are topological materials for systems with additional symmetries and in other dimensions. Whether a complete equivalence holds for those systems remains an open problem. We hope to extend our study to those systems in the future.

\section{ACKNOWLEDGMENTS}

We would like to thank Masatoshi Sato for his comments. This work is supported in part by the Japanese Grant-in-Aid for Scientific Research (No. 15K05054, No. 18H01216, No. 18H04484, and No. 18K03620).

\section{APPENDIX A: WARD-TAKAHASHI IDENTITIES}

In this appendix, we derive various identities among vertex functions and the inverse fermion propagator obtained from gauge invariance, i.e., Ward-Takahashi identities. The finite difference operator which appears in the hopping term of the lattice fermion system can be expressed in terms of infinite series of derivatives. For example, consider a gauge invariant fermion bilinear term connected by a straight line Wilson line in the $\mu$ direction as

$$
X \equiv \psi^{\dagger}(t, \vec{x}) e^{i \int_{\vec{x}}^{\vec{x}+a \vec{\mu}} d \vec{r}^{\prime} \cdot \vec{A}\left(\vec{r}^{\prime}\right)} \psi(t, \vec{x}+a \vec{\mu}),
$$

where $a$ is the lattice spacing and $\vec{\mu}$ is the unit vector in the $\mu$ direction. This term can be formally expanded as

$$
X=a \sum_{n=0}^{\infty} \psi^{\dagger}(t, \vec{x}) \sum_{n=0}^{\infty} \frac{a^{n}}{n !}\left(D_{\mu}^{n} \psi\right)(t, \vec{x}),
$$

where $D_{\mu}=\partial_{\mu}+i A_{\mu}$. We assume that the Hamiltonian can be expressed in terms of all sorts of fermion hopping terms connected by the Wilson lines of arbitrary contours or superpositions of them. Then, the action can be formally expanded as

$S=\int d t \sum_{\vec{x}} \sum_{n=0}^{\infty} \psi^{\dagger}(t, \vec{x}) M_{\mu_{1} \cdots \mu_{n}}\left(D_{\mu_{1}} \cdots D_{\mu_{n}} \psi\right)(t, \vec{x})$

where summation over $\mu_{1}, \ldots, \mu_{n}$ are implicit. $M_{\mu_{1} \cdots \mu_{n}}$ are some $N \times N$ matrix where $N=N_{c}+N_{v}$ is the number of fermion degrees of freedom per site. 
Expanding this action in terms of gauge fields and making Fourier transformations, one can obtain the formal expressions of the inverse propagator and the vertex functions in momentum space. In the following, let us denote the inverse fermion propagator with momentum $p$ as $S_{F}^{-1}(p)$ and the vertex functions with incoming fermion momentum $p$ and $n$ photons with incoming momentum $k_{i}$ and $\mu_{i}$ components $(i=1, \ldots, n)$ and outgoing fermion with momentum $p+\sum_{i=1}^{n} k_{i}$ as $\Gamma^{(n)}\left[k_{1}, \mu_{1} ; \cdots ; k_{n}, \mu_{n} ; p\right]$. Then the formal expression gives

$$
\begin{gathered}
S_{F}^{-1}(p)=\sum_{n=0}^{\infty} M_{\mu_{1} \cdots \mu_{n}} \prod_{i=1}^{n}\left(i p_{\mu_{i}}\right), \\
\Gamma^{(1)}[k, \mu ; p]=-i \sum_{n=1}^{\infty} \sum_{a=1}^{n} M_{\mu_{1} \cdots \mu_{a-1} \mu \mu_{a+1} \cdots \mu_{n}} \prod_{i=1}^{a-1}\left(i(p+k)_{\mu_{i}}\right) \prod_{i=a+1}^{n}\left(i p_{\mu_{i}}\right), \\
\Gamma^{(2)}[k, \mu ; l, \nu ; p]=-i^{2} \sum_{n=1}^{\infty} \sum_{a, b=1}^{n} M_{\mu_{1} \cdots \mu_{a-1} \mu \mu_{a+1} \cdots \mu_{b-1} \nu \mu_{b+1} \cdots \mu_{n}} \prod_{i=1}^{a-1}\left(i(p+k+l)_{\mu_{i}}\right) \prod_{i=a+1}^{b-1}\left(i(p+l)_{\mu_{i}}\right) \prod_{i=b+1}^{n}\left(i p_{\mu_{i}}\right) \\
-i^{2} \sum_{n=1}^{\infty} \sum_{a, b=1}^{n} M_{\mu_{1} \cdots \mu_{a-1} \nu \mu_{a+1} \cdots \mu_{b-1} \mu \mu_{b+1} \cdots \mu_{n}} \prod_{i=1}^{a-1}\left(i(p+k+l)_{\mu_{i}}\right) \prod_{i=a+1}^{b-1}\left(i(p+k)_{\mu_{i}}\right) \prod_{i=b+1}^{n}\left(i p_{\mu_{i}}\right), \\
\Gamma^{(3)}[k, \mu ; l, \nu, r, \lambda ; p]=-i^{3} \sum_{n=1}^{\infty} \sum_{a, b, c=1}^{n} M_{\mu_{1} \cdots \mu_{a-1} \mu \mu_{a+1} \cdots \mu_{b-1} \nu \mu_{b+1} \cdots \mu_{c-1} \lambda \mu_{c+1} \cdots \mu_{n}} \\
\prod_{a<b<c}^{a-1}\left(i(p+k+l+r)_{\mu_{i}}\right) \prod_{i=a+1}^{b-1}\left(i(p+l+r)_{\mu_{i}}\right) \prod_{i=b+1}^{c-1}\left(i(p+r)_{\mu_{i}}\right) \prod_{i=c+1}^{n}\left(i p+r_{\mu_{i}}\right) \\
+[\text { other } 5 \text { terms obtained from the permutaion of }(\mu, k),(\nu, l),(\lambda, r)] .
\end{gathered}
$$

Differentiating Eq. (A4) with respect to $p_{\mu}$ and taking the soft photon limit $(k \rightarrow 0)$ in Eq. (A5), one obtains

$$
\frac{\partial S_{F}^{-1}(p)}{\partial p_{\mu}}=-\left.\Gamma^{(1)}[k, \mu ; p]\right|_{k=0}=i \sum_{n=1}^{\infty} \sum_{a=1}^{n} M_{\mu_{1} \cdots \mu_{a-1} \mu \mu_{a+1} \cdots \mu_{n}} \prod_{i \neq a}^{1 \sim n}\left(i p_{\mu_{i}}\right) .
$$

This is the well-known Ward-Takahashi identity in QED, which is generalized for the lattice fermion system.

\section{First order Ward-Takahashi identities}

It is interesting to note that we could also obtain Ward-Takahashi identities for quantities involving higher order terms in photon momenta and multiphoton vertex functions. In order to see that, let us take the second derivatives of Eq. (A4). One obtains

$$
\frac{\partial^{2} S_{F}^{-1}(p)}{\partial p_{\mu} \partial p_{\nu}}=i^{2} \sum_{n=1}^{\infty} \sum_{\substack{a, b=1 \\ a<b}}^{n}\left[M_{\mu_{1} \cdots \mu_{a-1} \mu \mu_{a+1} \cdots \mu_{b-1} \nu \mu_{b+1} \cdots \mu_{n}}+(\mu \leftrightarrow \nu)\right] \prod_{i \neq a, b}^{1 \sim n}\left(i p_{\mu_{i}}\right) .
$$

Let us also differentiate Eq. (A5) with $k_{\nu}$ or $p_{\nu}$ and take the soft photon limit. We obtain

$$
\begin{gathered}
\left.\frac{\partial \Gamma^{(1)}[k, \mu ; p]}{\partial k_{\nu}}\right|_{k=0}=-i^{2} \sum_{n=1}^{\infty} \sum_{\substack{a, b=1 \\
a<b}}^{n} M_{\mu_{1} \cdots \mu_{a-1} \nu \mu_{a+1} \cdots \mu_{b-1} \mu \mu_{b+1} \cdots \mu_{n}} \prod_{i \neq a, b}^{1 \sim n}\left(i p_{\mu_{i}}\right) \\
\left.\frac{\partial \Gamma^{(1)}[k, \mu ; p]}{\partial p_{\nu}}\right|_{k=0}=-i^{2} \sum_{n=1}^{\infty} \sum_{\substack{a, b=1 \\
a<b}}^{n}\left[M_{\mu_{1} \cdots \mu_{a-1} \mu \mu_{a+1} \cdots \mu_{b-1} \nu \mu_{b+1} \cdots \mu_{n}}+(\mu \leftrightarrow \nu)\right] \prod_{i \neq a, b}^{1 \sim n}\left(i p_{\mu_{i}}\right) .
\end{gathered}
$$


Taking also the soft photon limit of Eq. (A6), we obtain

$$
\left.\Gamma^{(2)}[k, \mu ; l, \nu ; p]\right|_{k, l=0}=-i^{2} \sum_{n=1}^{\infty} \sum_{\substack{a, b=1 \\ a<b}}^{n}\left[M_{\mu_{1} \cdots \mu_{a-1} \mu \mu_{a+1} \cdots \mu_{b-1} \nu \mu_{b+1} \cdots \mu_{n}}+(\mu \leftrightarrow \nu)\right] \prod_{i \neq a, b}^{1 \sim n}\left(i p_{\mu_{i}}\right) .
$$

We then obtain the following identities:

$$
\frac{\partial^{2} S_{F}^{-1}(p)}{\partial p_{\mu} \partial p_{\nu}}=-\left.\left(\frac{\partial \Gamma^{(1)}[k, \mu ; p]}{\partial k_{\nu}}+(\mu \leftrightarrow \nu)\right)\right|_{k=0}=-\left.\frac{\partial \Gamma^{(1)}[k, \mu ; p]}{\partial p_{\nu}}\right|_{k=0}=-\left.\Gamma^{(2)}[k, \mu ; l, \nu ; p]\right|_{k, l=0} .
$$

\section{Second order Ward-Takahashi identities}

We could go even further in higher order. Taking the third derivative of Eq. (A4), we obtain

$$
\frac{\partial^{3} S_{F}^{-1}(p)}{\partial p_{\mu} \partial p_{\nu} \partial p_{\lambda}}=i^{3} \sum_{n=1}^{\infty} \sum_{\substack{a, b, c=1 \\ a<b<c}}^{n}\left[M_{\mu_{1} \cdots \mu_{a-1} \mu \mu_{a+1} \cdots \mu_{b-1} \nu \mu_{b+1} \cdots \mu_{c-1} \lambda \mu_{c+1} \cdots \mu_{n}}+(\text { perm. in }(\mu, \nu, \lambda))\right] \times \prod_{i \neq a, b, c}^{1 \sim n}\left(i p_{\mu_{i}}\right) .
$$

The second derivatives of Eq. (A5) with respect to $k$ or $p$ give

$$
\begin{gathered}
\left.\frac{\partial^{2} \Gamma^{(1)}[k, \mu ; p]}{\partial k_{\nu} \partial k_{\lambda}}\right|_{k=0}=-i^{3} \sum_{n=1}^{\infty} \sum_{\substack{a, b, c=1 \\
a<b<c}}^{n}\left[M_{\mu_{1} \cdots \mu_{a-1} \nu \mu_{a+1} \cdots \mu_{b-1} \lambda \mu_{b+1} \cdots \mu_{c-1} \mu \mu_{c+1} \cdots \mu_{n}}+(\nu \leftrightarrow \lambda)\right] \times \prod_{i \neq a, b, c}^{1 \sim n}\left(i p_{\mu_{i}}\right) \\
\left.\frac{\partial^{2} \Gamma^{(1)}[k, \mu ; p]}{\partial k_{\nu} \partial p_{\lambda}}\right|_{k=0}=-i^{3} \sum_{n=1}^{\infty} \sum_{\substack{a, b, c=1 \\
a<b<c}}^{n}\left[M_{\mu_{1} \cdots \mu_{a-1} \nu \mu_{a+1} \cdots \mu_{b-1} \lambda \mu_{b+1} \cdots \mu_{c-1} \mu \mu_{c+1} \cdots \mu_{n}}+(\nu \leftrightarrow \lambda)+((\nu, \lambda, \mu) \leftrightarrow(\nu, \mu, \lambda))\right] \prod_{i \neq a, b, c}^{1 \sim n}\left(i p_{\mu_{i}}\right) \\
\left.\frac{\partial^{2} \Gamma^{(1)}[k, \mu ; p]}{\partial p_{\nu} \partial p_{\lambda}}\right|_{k=0}=-i^{3} \sum_{n=1}^{\infty} \sum_{\substack{a, b, c=1 \\
a<b<c}}^{n}\left[M_{\mu_{1} \cdots \mu_{a-1} \mu \mu_{a+1} \cdots \mu_{b-1} \nu \mu_{b+1} \cdots \mu_{c-1} \lambda \mu_{c+1} \cdots \mu_{n}}+(\operatorname{perm} . \operatorname{in}(\mu, \nu, \lambda))\right] \times \prod_{i \neq a, b, c}^{1 \sim n}\left(i p_{\mu_{i}}\right) .
\end{gathered}
$$

From the first derivative of Eq. (A6) with respect to $k$ or $l$ or $p$, we obtain

$$
\begin{aligned}
&\left.\frac{\partial \Gamma^{(2)}[k, \mu ; l, \nu ; p]}{\partial k_{\lambda}}\right|_{k, l=0}=-i^{3} \sum_{n=1}^{\infty} \sum_{\substack{a, b, c=1 \\
a<b<c}}^{n}\left[M_{\mu_{1} \cdots \mu_{a-1} \lambda \mu_{a+1} \cdots \mu_{b-1} \mu \mu_{b+1} \cdots \mu_{c-1} \nu \mu_{c+1} \cdots \mu_{n}}\right. \\
&+((\lambda, \mu, \nu) \leftrightarrow(\lambda, \nu, \mu))+((\lambda, \mu, \nu) \leftrightarrow(\nu, \lambda, \mu))] \prod_{i \neq a, b, c}^{1 \sim n}\left(i p_{\mu_{i}}\right), \\
&\left.\frac{\partial \Gamma^{(2)}[k, \mu ; l, \nu ; p]}{\partial l_{\lambda}}\right|_{k, l=0}=-i^{3} \sum_{n=1}^{\infty} \sum_{\substack{a, b, c=1 \\
a<b<c}}^{n}\left[M_{\mu_{1} \cdots \mu_{a-1} \lambda \mu_{a+1} \cdots \mu_{b-1} \mu \mu_{b+1} \cdots \mu_{c-1} \nu \mu_{c+1} \cdots \mu_{n}}\right. \\
&+((\lambda, \mu, \nu) \leftrightarrow(\mu, \lambda, \nu))+((\lambda, \mu, \nu) \leftrightarrow(\lambda, \nu, \mu))] \prod_{i \neq a, b, c}^{1 \sim n}\left(i p_{\mu_{i}}\right), \\
&\left.\frac{\partial \Gamma^{(2)}[k, \mu ; l, \nu ; p]}{\partial p_{\lambda}}\right|_{k, l=0}=-i^{3} \sum_{n=1}^{\infty} \sum_{\substack{a, b, c=1 \\
a<b<c}}^{n}\left[M_{\mu_{1} \cdots \mu_{a-1} \mu \mu_{a+1} \cdots \mu_{b-1} \nu \mu_{b+1} \cdots \mu_{c-1} \lambda \mu_{c+1} \cdots \mu_{n}}+(\operatorname{perm} . \operatorname{in}(\mu, \nu, \lambda))\right] \prod_{i \neq a, b, c}^{1 \sim n}\left(i p_{\mu_{i}}\right) .
\end{aligned}
$$


These equations give the following identities:

$$
\begin{aligned}
\left.\frac{\partial^{2} \Gamma^{(1)}[k, \mu ; p]}{\partial k_{\nu} \partial p_{\lambda}}\right|_{k=0} & =\left.\frac{\partial \Gamma^{(2)}[k, \mu ; l, \lambda ; p]}{\partial k_{\nu}}\right|_{k, l=0} \\
& =\left.\frac{\partial \Gamma^{(2)}[k, \lambda ; l, \mu ; p]}{\partial l_{\nu}}\right|_{k, l=0}
\end{aligned}
$$

\section{Third order Ward-Takahashi identities}

Carrying out similar calculations by simply differentiating $\Gamma^{(2)}$ and $\Gamma^{(3)}$ given in Eqs. (A6) and (A7), we can see that the following identity holds:

$$
\left.\frac{\partial^{2} \Gamma^{(3)}[q, \mu ; r, \nu ; s, \lambda ; p]}{\partial q_{\alpha} \partial r_{\beta}}\right|_{q, r, s=0}=\left.\frac{\partial^{3} \Gamma^{(2)}[q, \mu ; r, \nu ; p]}{\partial q_{\alpha} \partial r_{\beta} \partial p_{\lambda}}\right|_{q, r=0} .
$$

\section{APPENDIX B: USEFUL FORMULAS FOR ENERGY EIGENSTATE}

Consider a Hamiltonian in momentum representation $H(p)$ and the normalized complete set of eigenstates at momentum $p$ labeled by index $\alpha$ ( $\alpha$ runs over the states in both the valence and the conduction bands) with the following properties:

$$
\begin{gathered}
H(p)|\alpha(p)\rangle=E_{\alpha}(p)|\alpha(p)\rangle, \\
\langle\alpha \mid \beta\rangle=\delta_{\alpha \beta}, \\
\langle\alpha(p)|H(p)| \beta(p)\rangle=E_{\alpha}(p) \delta_{\alpha \beta}, \quad(\alpha, \beta=1, \ldots, N) .
\end{gathered}
$$

Let us consider differentiation with respect to $p_{\mu}$. Here we introduce the simplified notation

$$
\left|\partial_{\mu} \alpha\right\rangle=\partial_{\mu}|\alpha\rangle \equiv \frac{\partial}{\partial p_{\mu}}(|\alpha(p)\rangle), \quad \partial_{\mu} H \equiv \frac{\partial}{\partial p_{\mu}}(H(p)) .
$$

Then differentiating Eq. (B2), we obtain

$$
\left\langle\partial_{\mu} \alpha \mid \beta\right\rangle=-\left\langle\alpha \mid \partial_{\mu} \beta\right\rangle \text {. }
$$

Also, differentiating Eq. (B3) and making a little algebra, we have

$$
\left(E_{\alpha}-E_{\beta}\right)\left\langle\alpha \mid \partial_{\mu} \beta\right\rangle+\left\langle\alpha\left|\partial_{\mu} H\right| \beta\right\rangle=\partial_{\mu} E_{\alpha} \delta_{\alpha \beta} .
$$

This means the matrix element of the momentum derivative of the Hamiltonian is given as

$$
\begin{gathered}
\left\langle\alpha\left|\partial_{\mu} H\right| \alpha\right\rangle=\partial_{\mu} E_{\alpha}, \\
\left\langle\alpha\left|\partial_{\mu} H\right| \beta\right\rangle=-\left(E_{\alpha}-E_{\beta}\right)\left\langle\alpha \mid \partial_{\mu} \beta\right\rangle \quad(\alpha \neq \beta) .
\end{gathered}
$$

\section{Degenerate flat band case}

Let us now consider the special case where all the energies in the valence bands and those in the conduction bands are degenerate and flat. Then, one can easily see that

$$
\begin{gathered}
\left\langle a(\vec{p})\left|\partial_{\mu} H(\vec{p})\right| b(\vec{p})\right\rangle=0, \\
\left\langle\dot{a}(\vec{p})\left|\partial_{\mu} H(\vec{p})\right| \dot{b}(\vec{p})\right\rangle=0, \\
\left\langle a(\vec{p})\left|\partial_{\mu} H(\vec{p})\right| \dot{b}(\vec{p})\right\rangle=\left(E_{c}-E_{v}\right)\left\langle a \mid \partial_{\mu} \dot{b}\right\rangle,
\end{gathered}
$$

$\left\langle\dot{a}(\vec{p})\left|\partial_{\mu} H(\vec{p})\right| b(\vec{p})\right\rangle=-\left(E_{c}-E_{v}\right)\left\langle\dot{a} \mid \partial_{\mu} b\right\rangle$,

$$
\left(a, b=1, \ldots, N_{v}, \dot{a}, \dot{b}=1, \ldots, N_{c}\right),
$$

where the states with undotted indices $|a\rangle,|b\rangle$ are in the valance bands with constant energy $E_{v}<0$ and those with dotted indices $|\dot{a}\rangle,|\dot{b}\rangle$ are in the conduction bands with constant energy $E_{c}>0$.

Let us define the Berry connection using the negative energy eigenstates as

$$
\mathcal{A}^{a b} \equiv \mathcal{A}_{\mu}^{a b} d x^{\mu}=-i\left\langle a \mid \partial_{\mu} b\right\rangle d x^{\mu} \equiv-i\langle a \mid d b\rangle .
$$

Then the Berry curvature $\mathcal{F}^{a b}$ is

$$
\begin{aligned}
\mathcal{F}^{a b}= & (d \mathcal{A}+i \mathcal{A \mathcal { A }})^{a b}=-i\langle d a \mid d b\rangle \\
& +i \sum_{c=1}^{N_{v}}(-i)\langle a \mid d c\rangle(-i)\langle c \mid d b\rangle \\
= & -i\left[\sum_{c=1}^{N_{v}}\langle d a \mid c\rangle\langle c \mid d b\rangle+\sum_{\dot{c}=1}^{N_{c}}\langle d a \mid \dot{c}\rangle\langle\dot{c} \mid d b\rangle\right] \\
& -i \sum_{c=1}^{N_{v}}\langle a \mid d c\rangle\langle c \mid d b\rangle \\
= & \left.i \sum_{c=1}^{N_{v}}\langle a \mid d c\rangle\langle c \mid d b\rangle+i \sum_{\dot{c}=1}^{N_{c}}\langle a \mid d \dot{c}\rangle\langle\dot{c} \mid d b\rangle\right) \\
& -i \sum_{c=1}^{N_{v}}\langle a \mid d c\rangle\langle c \mid d b\rangle \\
= & i \sum_{\dot{c}=1}^{N_{c}}\langle a \mid d \dot{c}\rangle\langle\dot{c} \mid d b\rangle .
\end{aligned}
$$

\section{APPENDIX C: USEFUL FORMULAS FOR $p^{0}$ INTEGRATION}

We define the following $p^{0}$ integrations:

$$
I^{[m, n]}\left(E_{1}, E_{2}\right) \equiv \int \frac{d p^{0}}{2 \pi} \frac{1}{\left(i p^{0}+E_{1}\right)^{m}\left(i p^{0}+E_{2}\right)^{n}}\left(E_{1} \neq E_{2}\right) .
$$


Simple contour integral by adding a contour in the lower half semi-circle in the complex $p^{0}$ plane and picking up poles in the lower half plane, we obtain

$I^{[m, n]}\left(E_{1}, E_{2}\right)=(-1)^{m} \frac{(m+n-2) !}{(m-1) !(n-1) !} \cdot \frac{\theta\left(-E_{1}\right)-\theta\left(-E_{2}\right)}{\left(-E_{1}+E_{2}\right)^{m+n-1}}$.
In particular, when $(m, n)=(n+1, n)$

$I^{[n+1, n]}\left(E_{1}, E_{2}\right)=(-1)^{n+1} \frac{(2 n) !}{2(n !)^{2}} \cdot \frac{\theta\left(-E_{1}\right)-\theta\left(-E_{2}\right)}{\left(-E_{1}+E_{2}\right)^{2 n}}$

holds. This formula is useful in making $p^{0}$ integration of the propagator expressions for the Chern-Simons level.
[1] D. J. Thouless, M. Kohmoto, M. P. Nightingale, and M. den Nijs, Quantized Hall Conductance in a TwoDimensional Periodic Potential, Phys. Rev. Lett. 49, 405 (1982).

[2] Q. Niu, D. J Thouless, and Y.-S. Wu, Quantized hall conductance as a topological invariant, Phys. Rev. B 31, 3372 (1985).

[3] K. Ishikawa, Chiral Anomaly and Quantized Hall Effect, Phys. Rev. Lett. 53, 1615 (1984).

[4] K. Ishikawa, Axial anomaly in three-dimensions and planar fermions, Phys. Rev. D 31, 1432 (1985).

[5] K. Ishikawa and T. Matsuyama, Magnetic field induced multi component QED in three dimensions and quantum Hall effect, Z. Phys. C 33, 41 (1986).

[6] K. Ishikawa and T. Matsuyama, A microscopic theory of the quantum Hall effect, Nucl. Phys. B280, 523 (1987).

[7] H. So, Induced Chern-Simons class with lattice fermions, Prog. Theor. Phys. 73, 528 (1985).

[8] H. So, Induced topological invariants by lattice fermions in odd dimensions, Prog. Theor. Phys. 74, 585 (1985).
[9] M. F. L. Golterman, K. Jansen, and D. B. Kaplan, ChernSimons currents and chiral fermions on the lattice, Phys. Lett. B 301, 219 (1993).

[10] D. B. Kaplan, A method for simulating chiral fermions on the lattice, Phys. Lett. B 288, 342 (1992).

[11] K. Jansen, Chiral fermions and anomalies on a finite lattice, Phys. Lett. B 288, 348 (1992).

[12] K. Jansen and M. Schmaltz, Critical momenta of lattice chiral fermions, Phys. Lett. B 296, 374 (1992).

[13] D. B. Kaplan and M. Schmaltz, Domain wall fermions and the eta invariant, Phys. Lett. B 368, 44 (1996).

[14] E. Witten, Three lectures on topological phases of matter, La Rivista del Nuovo Cimento (2016).

[15] M. A. Zubkov, Wigner transformation, momentum space topology, and anomalous transport, Ann. Phys. (Amsterdam) 373, 298 (2016).

[16] X.-L. Qi, T. Hughes, and S.-C. Zhang, Topological field theory of time-reversal invariant insulators, Phys. Rev. B 78, 195424 (2008).

[17] R. Peierls, On the theory of diamagnetism of conduction electrons, Z. Phys. 80, 763 (1933). 